\title{
Performance Evaluation of 802.15.3a UWB Channel Model with Antipodal, Orthogonal and DPSK Modulation Scheme
}

\author{
Sachin Taran ${ }^{\mathrm{a}}$, Dhiraj Nitnaware ${ }^{\mathrm{b}}$ \\ ${ }^{a}$ Electronics and Communication, MGI, Indore, India \\ ${ }^{b}$ Electronics and Communication, IET DAVV, Indore, India
}

\begin{abstract}
In this paper, we have investigated the performance of IEEE 802.15.3a channel model with different modulation scheme. The performance parameter Average Bit Error Rate (ABER) is selected. We find out the BER performance of UWB system over the 802.15.3a indoor channel model for all channels profile CM1, CM2, CM3 and CM4 with different modulation scheme. In this paper, all modulation scheme used in UWB are included in different main categories. The major categories are synchronous and asynchronous. In synchronous mode, we again divided different modulation scheme e.g. BPSK, PPM, PAM etc into antipodal and orthogonal schemes. In the asynchronous category, we have studied about DPSK. All these modulation categories are comparing on the common platform and finally we got antipodal modulation scheme category is good for UWB in term of ABER.
\end{abstract}

Index Terms: UWB (Ultra Wide Band), log normal fading, Wilkinson Method, 802.15.3a channel model, Antipodal signalling, and orthogonal signalling.

(C) 2016 Published by MECS Publisher. Selection and/or peer review under responsibility of the Research Association of Modern Education and Computer Science

\section{Introduction}

A widely accepted UWB (Ultra Wide Band) indoor communication channel model 802.15.3a developed by channel modelling subcommittee and it is define in [1,2].According to [2], the 802.15.3a channel model UWB system exhibits high frequency selective fading and can be modelled as a discrete linear filter whose impulse response is expressed as,

$$
\mathrm{h}(\mathrm{t})=\sum_{\mathrm{l}=0}^{\mathrm{L}_{\max }-1} \alpha_{l} \delta\left(\mathrm{t}-\tau_{\mathrm{l}}\right)
$$

\footnotetext{
* Corresponding author
}

E-mail address: 
The $\mathrm{L}_{\max }$ is the maximum number of resolvable multipath components (MPCs) and it is decided by the number of fingers of rake receiver or sampling resolution of receiver. The $\alpha_{l}$ is the channel fading coefficient for the $l^{\text {th }}$ path and its modelled as lognormal random variable, whose parameter depends on the arrival time $\tau_{l}$. The $\tau_{l}$ is arrival time of $l^{\text {th }}$ path relative to $1^{\text {st }}$ path $\left(l=0 \& \tau_{0}=0\right.$ assumed $)$ and $\delta(\mathrm{t})$ is Dirac delta function.

The channel gain $\alpha_{l}$ is modelled as $\alpha_{l}=p_{l} \beta_{l}$, where $p_{l}=+1 /-1$ with equal probability it models the random pulse inversion that can occur due to reflections it is define in [2]. The second term $\beta_{l}$ is a magnitude of $l^{\text {th }}$ MPC and it is log normally distributed for indoor channels. The parameters of lognormal random variable are mean and standard deviation. The standard deviation of fading amplitude is in the range of 3-5dB given in [2] and mean value depends on the parameters of channel profile CM1, CM2, CM3, CM4 such as cluster arrival rate , ray arrival rate ,cluster decay factor, ray decay factor etc given by [1,2]. These channel profile parameters such as distribution of path arrival time sequence $\tau_{l}$ and power delay profile (PDP) of the channel are chosen to follow the modified Saleh-Valenzuela (S-V) model suggested in [3].

Effect of different modulation scheme on 802.15.3a channel model is also studied by so many researchers but in that research some of them not studied all modulation categories just like [4]. In this paper the modelled UWB channel model very well but they only study about hybrid combination of pulse amplitude and pulse position modulation not about the other better options. In [5] they studied about antipodal and orthogonal signalling but there analytical method is not accurate because they not modelled UWB channel accurately. So there frame work cannot be achieved desire accuracy. In this paper we proposed a analytical method that cover all modulation scheme which is used in UWB and we also modelled UWB channel model accurately with the help of Wilkinson's method.

\section{Problem Formation}

As in [6] they present the instantaneous signal to noise ratio (SNR) model by considering one of the channel impairments i.e. intra pulse interference (IPI) according to [6] for a realistic UWB system having a finite temporal resolution $T_{p}$ i.e. often limited by the receiver sampling frequency or the width of UWB pulse. The instantaneous signal to noise ratio (SNR) per bit can be written as

$$
\gamma_{i}=\frac{\mathrm{E}_{S}}{\mathrm{~N}_{0}} \sum_{l=0}^{\left[\frac{\mathrm{T}_{\mathrm{i}}}{\mathrm{T}_{\mathrm{p}}}\right]-1} \alpha_{l}^{2}=\frac{\mathrm{E}_{S}}{\mathrm{~N}_{0}} \sum_{l=0}^{\left[\frac{\mathrm{T}_{\mathrm{i}}}{\mathrm{T}_{\mathrm{p}}}\right]-1}\left[\sum_{\mathrm{i} \in \mathrm{I}_{l}}\left(\beta_{\mathrm{i}} \mathrm{P}_{\mathrm{i}}\right)\right]^{2}
$$

In this model second summation is taken for accounting the intra pulse interference i.e. occurs between MPCs. where $\mathrm{I}_{l}$ is set given by: $\mathrm{I}_{l}=\left\{\mathrm{i} \mid l T_{p} \leq \tau_{i}<(l+1) T_{p}\right\}$ denotes the collection of multipath components (MPCs) arriving in the interval $\left[l T_{p},(l+1) T_{p}\right]$, i.e. the number of MPCs in a single finite temporal resolution but right now in this paper the impairments that we want to model is shadowing not the intra pulse interference so width of pulse is adjusted such that $T_{p}<<1$. In this case $\gamma_{l} \rightarrow \beta_{l} \alpha_{l}$ and equation (2) from the [6] can be written as

$$
\gamma_{i}=\frac{E_{s}}{\mathrm{~N}_{0}} \sum_{\mathrm{l}=0}^{\mathrm{L}_{\mathrm{max}}-1} \beta_{l}^{2}
$$

\section{Performance Analysis}

The major parameters that researchers generally use to figure out the performance of UWB channel are outage probability and average bit error rate (ABER). In this paper we are using the ABER.

The process of finding the ABER given in [7], the average bit error rate can be calculated by averaging the conditional BER which is $P\left(\gamma_{i}\right)$ over the probability density function (PDF) of instantaneous SNR i.e. $f\left(\gamma_{i}\right)$ as 


$$
P_{e}=\int_{0}^{\infty} P\left(\gamma_{i}\right) f\left(\gamma_{i}\right) d \gamma_{i}
$$

The conditional BER for fixed set of $\beta_{l}$ for different modulation scheme are given in table 1 .

\subsection{Data Modulation}

A number of modulation schemes may be used with UWB systems. The potential modulation schemes include both orthogonal and antipodal schemes.

\subsubsection{Antipodal Scheme (BPSK, PAM)}

The antipodal signal scheme in which multiplication of two transmitted symbol is -1.The classic binary pulse amplitude modulation (PAM) can be presented using e.g. two antipodal Gaussian pulses as shown in Figure 1 from [8].

The transmitted binary baseband pulse amplitude modulated information signal $(\mathrm{t})$ can be presented as

$$
\mathrm{X}(\mathrm{t})=d_{j} * \mathrm{P}(\mathrm{t})
$$

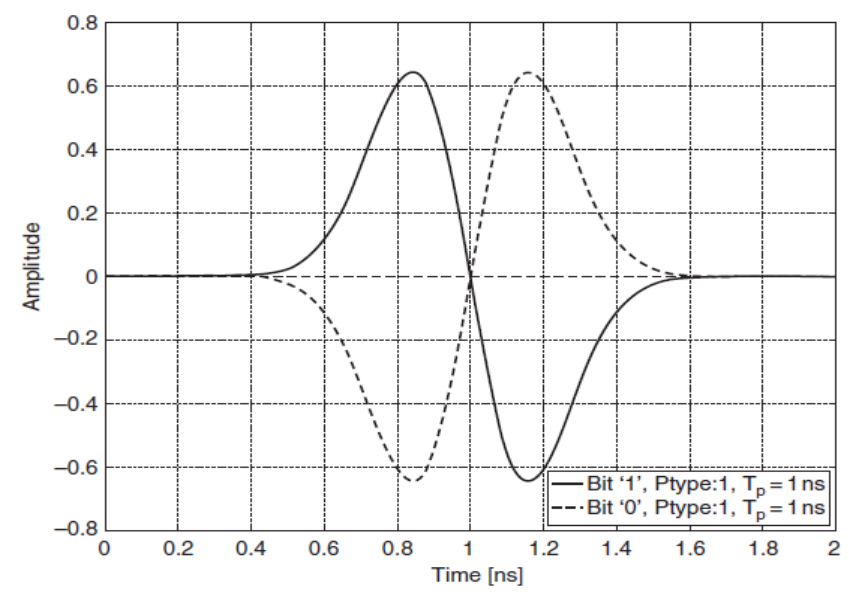

Fig.1. BPAM Pulse Shapes for ' 1 ' and '0' Bits

Where $P(t)$ represents the UBW pulse waveform, $\mathrm{j}$ represents the bit transmitted (' 0 ' or ' 1 ') and

$$
d_{j}= \begin{cases}-1, & j=0 \\ 1, & j=1\end{cases}
$$

\subsubsection{Orthogonal Scheme (OOK, PPM)}

The orthogonal signalling scheme in which multiplication of two transmitted symbol is 0 . The example of orthogonal modulation scheme is binary on-off keying (OOK). Using the above definitions of orthogonal modulation scheme with a single Gaussian pulse shown in figure 2, from [8]. 


$$
d_{j}= \begin{cases}0, & j=0 \\ 1, & j=1\end{cases}
$$

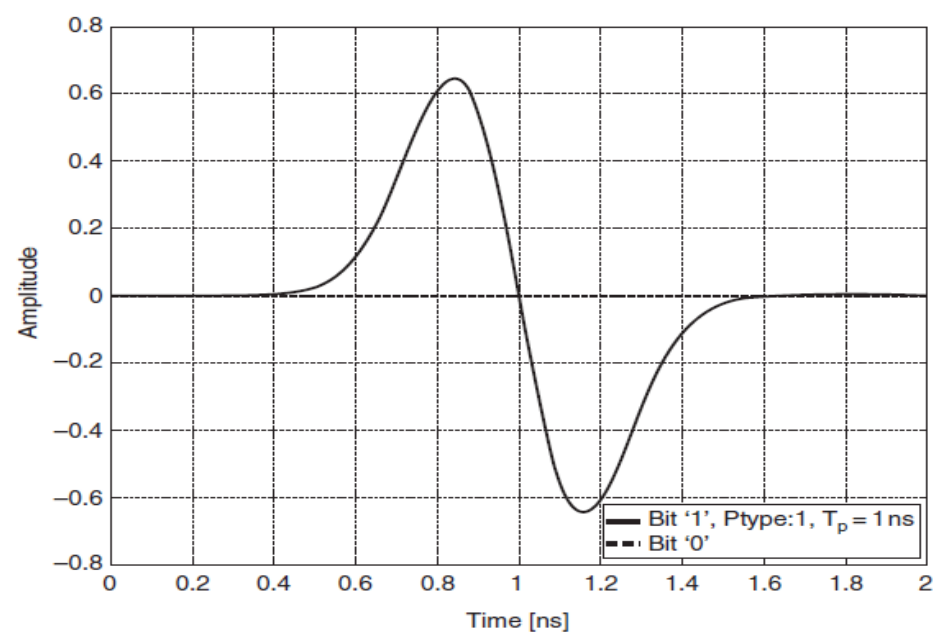

Fig.2. OOK Pulse Shapes for ' 1 ' and '0' Bits

\subsubsection{Asynchronous Modulation Scheme (DPSK)}

To solve the problem of synchronous receiver we can use differential detection technique. For binary phase shift keying this technique is called differential phase shift keying (DPSK). This problem can be solves by using encoding technique.

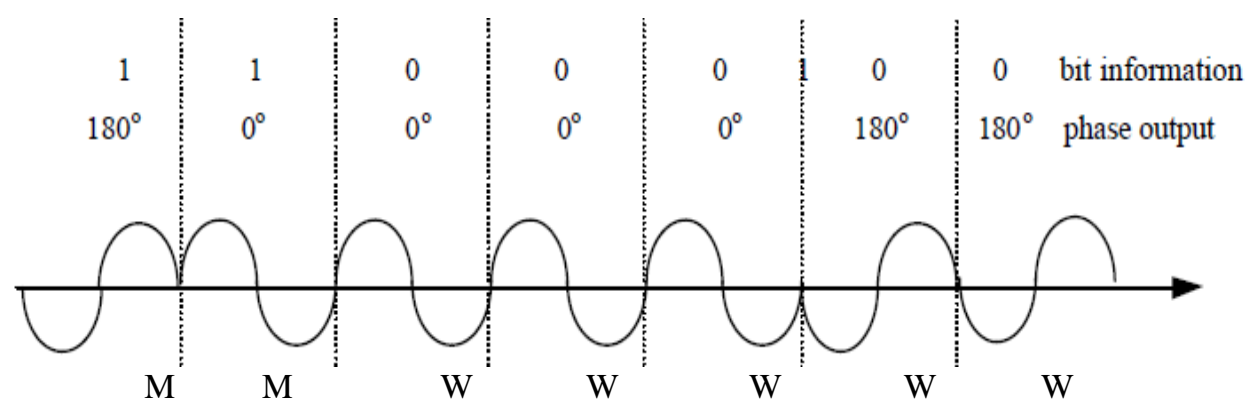

Fig.3. Phase Shifting and Waveform Output of Binary DPSK.

In fig. 3 taken from [9], high state is represented by an $M$ in modulated signal and low state is represented by W in modulated signal DPSK encodes two distinct signals of same frequency with 180 degree phase difference between the two.

\section{AWGN Effect On All Modulation Scheme Performance and PDF Calculation}


The conditional BER for all modulation schemes are given in table 1, by [8]. In each case conditional BER is a function of SNR.

Table 1. Binary Data Mapping Formats and BER Performances

\begin{tabular}{cc}
\hline Modulation Scheme & $\boldsymbol{P}\left(\boldsymbol{\gamma}_{i}\right)=$ Conditional BER \\
\hline BPSK (Antipodal) & $\mathrm{Q}\left(\sqrt{\frac{2 \mathrm{E}_{\mathrm{b}}}{N_{0}}}\right)=\mathrm{Q}(\sqrt{2 S N R})$ \\
PPM (Orthogonal) & $\mathrm{Q}\left(\sqrt{\frac{\mathrm{E}_{\mathrm{b}}}{N_{0}}}\right)=\mathrm{Q}(\sqrt{S N R})$ \\
OOK (Orthogonal) & $\mathrm{Q}\left(\sqrt{\frac{\mathrm{E}_{\mathrm{b}}}{N_{0}}}\right)=\mathrm{Q}(\sqrt{S N R})$ \\
DPSK (Asynchronous) & $\frac{1}{2} \exp \left(-\frac{\mathrm{E}_{\mathrm{b}}}{N_{0}}\right)=\frac{1}{2} \exp (-S N R)$ \\
\hline
\end{tabular}

For calculating the ABER we require the PDF of instantaneous SNR $f\left(\gamma_{i}\right)$. There are many methods for calculating the PDF of instantaneous SNR. A major section of research in the field of UWB has been done for finding the accurate PDF of their instantaneous SNR model. In [6] they have suggested three methods for approximating the PDF of instantaneous SNR model according to severances of fading. The distributions used in [6] to approximate the PDF of $\gamma_{i}$ are coxian, Lognormal, Gamma and parameters of these approximation are find out using least square error criteria by minimizing the mean square error. Our process for finding the PDF of instantaneous SNR is little bit different and it is best according to our model. In this paper we have computed the PDF of term $\left(\frac{E_{s}}{N_{0}}\right) \sum_{l=0}^{\mathrm{L}_{m a x}{ }^{-1}} \alpha_{l}{ }^{2}$. This term is simply the square of sum of lognormal random variables and estimation of distributions can be given by [9]. They have suggested three methods for the estimation of distribution. The method which we are using in this paper is Wilkinson's method.

Let assume that

$$
\gamma_{i}=\left(\frac{\mathrm{E}_{s}}{\mathrm{~N}_{0}}\right) \sum_{\mathrm{l}=0}^{\mathrm{L}_{\mathrm{max}}-1} \alpha_{l}^{2}=\sum_{\mathrm{l}=0}^{\mathrm{L}_{\mathrm{max}}-1} \mathrm{~g}_{l}
$$

Where $g_{l}=\left(\frac{E_{s}}{N_{0}}\right) \beta_{l}{ }^{2}$ and $\alpha_{l}=p_{l} \beta_{l}$. According to UWB channel committee report $\beta_{\mathrm{l}}$ is a lognormal random variable so $\mathrm{g}_{l}=\left(\frac{\mathrm{E}_{S}}{\mathrm{~N}_{0}}\right) \beta_{l}{ }^{2}$ is also lognormal RV. Thus $\gamma_{1}$ is a sum of L independent lognormal RVs.

Let a lognormal $\mathrm{RV} \beta_{l}=e^{m_{l}}$ where $m_{l}$ is a normal RV, i.e. $m_{l} \sim N\left(\mu_{m_{l}}, \sigma_{m_{l}}^{2}\right)$.

Then $\mathrm{g}_{l}=e^{a_{0}+2 m_{l}}$, where $a_{0}=\ln \left(\frac{\mathrm{E}_{s}}{\mathrm{~N}_{0}}\right)$.

The $\mathbf{J}^{\text {th }}$ moment of $\beta_{l}$ is given as

$$
\mathrm{E}\left\{\beta_{l}{ }^{j}\right\}=e^{\frac{j \mu_{m_{l}}+j^{2} \sigma_{m}^{2}}{2}}
$$

The PDF of sum of lognormal R.V. suggested by [9]. This procedure is based on the assumption that a sum of independent lognormal RV's is again a lognormal RV.

Let $\gamma_{i}=e^{z}$, where $z \sim N\left(\mu_{z}, \sigma_{z}^{2}\right)$, is a normal RV. In Wilkinson's method, the two parameter $\mu_{z}$ and $\sigma_{z}$ are obtained by matching the first two moments of $\gamma_{1}$ with the first two moments of $\sum_{\mathrm{l}=0}^{\mathrm{L}_{\mathrm{max}}{ }^{-1}} \mathrm{~g}_{l}$. These two parameters given as 


$$
\mu_{\mathrm{z}}=\ln \left(\frac{\mathrm{W}_{\mathrm{L} 1}^{2}}{\sqrt{\mathrm{W}_{\mathrm{L} 2}}}\right), \sigma_{\mathrm{z}}=\ln \left(\frac{\mathrm{W}_{\mathrm{L} 2}}{\mathrm{~W}_{\mathrm{L} 1}^{2}}\right)
$$

Where $\mathrm{W}_{\mathrm{L} 1}$ and $\mathrm{W}_{\mathrm{L} 2}$ are related to $\mu_{\mathrm{y}_{1}}$ and $\sigma_{\mathrm{y}_{1}}^{2}$ as

$$
\mathrm{W}_{\mathrm{L} 1}=\sum_{l=0}^{L-1} e^{\left(c_{0}+2 \mu_{m_{l}}+2 \sigma_{m_{l}}^{2}\right)}
$$

$\mathrm{W}_{\mathrm{L} 2}=\sum_{l=0}^{L-1} e^{2\left(c_{0}+2 \mu_{m_{l}}+4 \sigma_{m_{l}}^{2}\right)}+2 \sum e^{2\left(c_{0}+2 \mu_{y_{m}}+\mu_{y_{n}}+\sigma_{y_{m}}^{2}+\sigma_{y_{n}}^{2}\right)}$

Where the $2^{\text {nd }}$ sum in (6) is extended to all combinations of $(m, n), m<n=0 \ldots \mathrm{L}-1$.

The approximated PDF of $\gamma_{i}$ is given as

$$
f\left(\gamma_{i}\right)=\frac{1}{\gamma_{1} \sqrt{2 \pi \sigma_{z}^{2}}} \exp \left[-\frac{\left(\ln \left(\gamma_{1}\right)-\mu_{z}\right)^{2}}{2 \sigma_{Z}^{2}}\right]
$$

\section{Numerical Results: Framework Validation}

In these result we compare three different category of modulation schemes i.e. antipodal, orthogonal and one of asynchronous category i.e. DPSK on same platform. To obtain these results the number of fingers taken for rake receiver is ten and range of $\operatorname{SNR}\left(\frac{E_{S}}{N_{0}}\right)$ is 1 to $10 \mathrm{~dB}$.

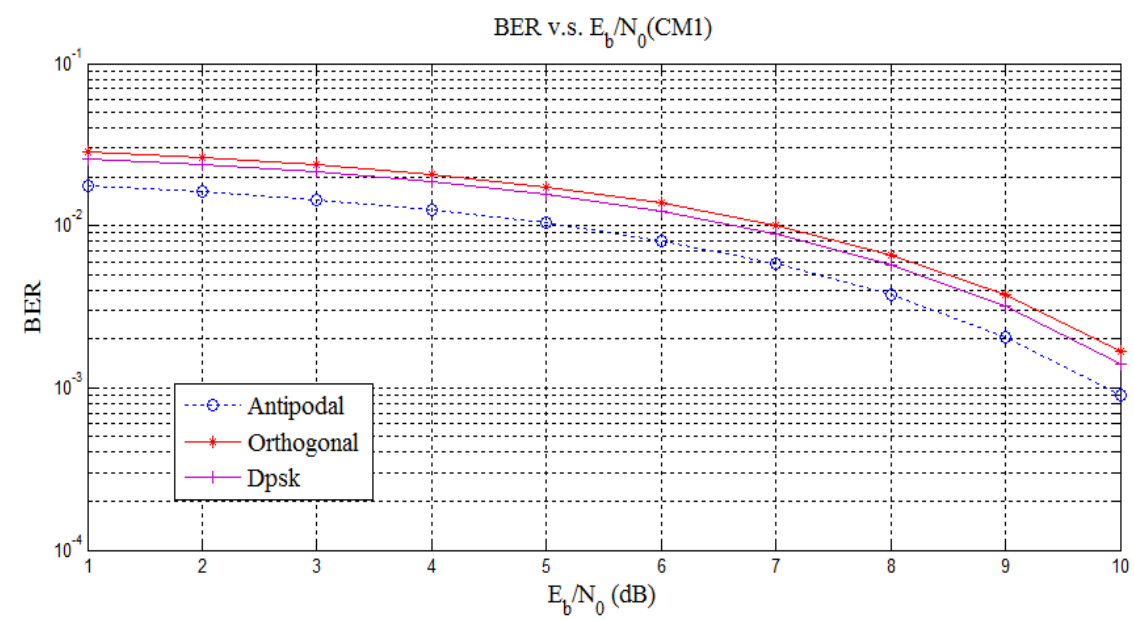

Fig.4. BER Performance of the IEEE 802.15.3a UWB Channel for CM1 with Different Modulation Scheme.

The above result is obtained for channel model 1. This channel model is of a line of sight (LOS) case with the transmitter and the receiver antenna being separated by a distance in the range (0-4 m). The parameters used for these result are given in [3].

From the figure 4 it is clear that the average bit error rate (ABER) for all modulation schemes in case of CM1 is near about $10^{-3}$ at SNR value of $10 \mathrm{~dB}$. 


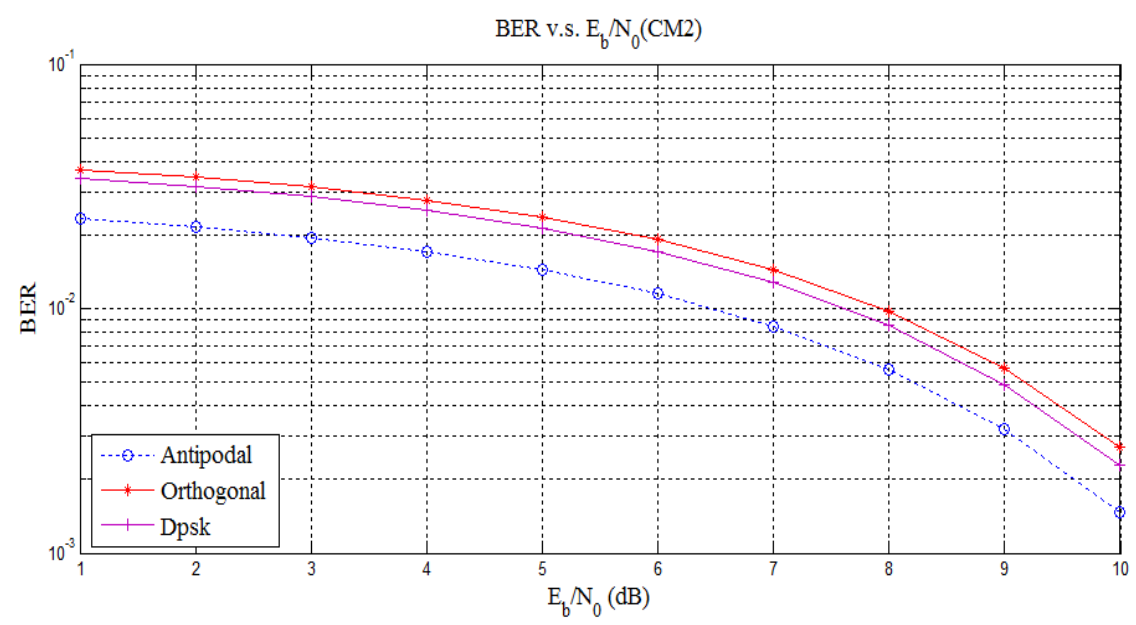

Fig.5. BER Performance of the IEEE 802.15.3a UWB Channel for CM2with Different Modulation Scheme.

The above result is obtained for channel model 2. This channel is a model for a non line of sight (NLOS) case with antenna separation being in the range (0-4 m). The parameters used for these result are given in [3].

From the figure 5 it is clear that the average bit error rate (ABER) for all modulation schemes in case of $\mathrm{CM} 2$ is above of $10^{-3}$ at $\mathrm{SNR}$ value of $10 \mathrm{~dB}$.

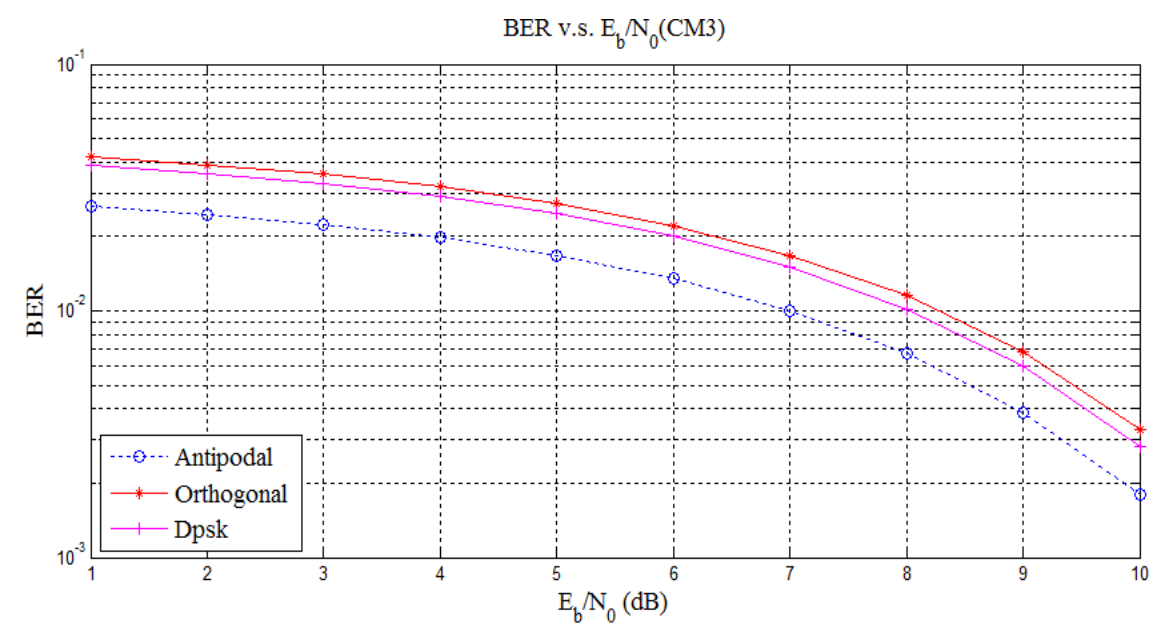

Fig.6. BER Performance of the IEEE 802.15.3a UWB Channel for CM3 with Different Modulation Scheme.

The above result is obtained for channel model3. This channel is a model for a non line of sight (NLOS) case with antenna separation being in the range (4-10 m). The parameters used for these result are given in [3].

From the figure it is clear that the average bit error rate (ABER) for all modulation schemes in case of CM3 is in the mid of $10^{-2}$ and $10^{-3}$ at SNR value of $10 \mathrm{~dB}$.

The below result is obtained for channel model 4. This channel is a model for a extreme NLOS multipath channel to fit a $25 \mathrm{~ns}$ RMS delay spread. The parameters used for these result are given in [3].

From the figure it is clear that the average bit error rate (ABER) for all modulation schemes in case of CM4 is closer to $10^{-2}$ at $\mathrm{SNR}$ value of $10 \mathrm{~dB}$. 


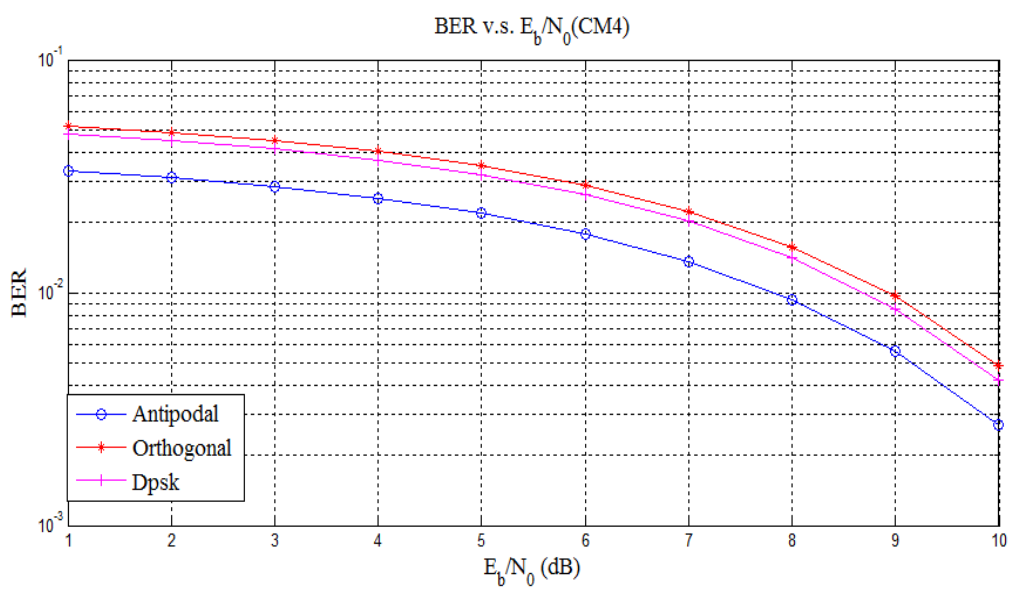

Fig.7. BER Performance of the IEEE 802.15.3a UWB Channel for CM4 with Different Modulation Scheme.

In all channel models minimum ABER is obtained for antipodal modulation scheme and maximum ABER is obtained for DPSK modulation scheme.

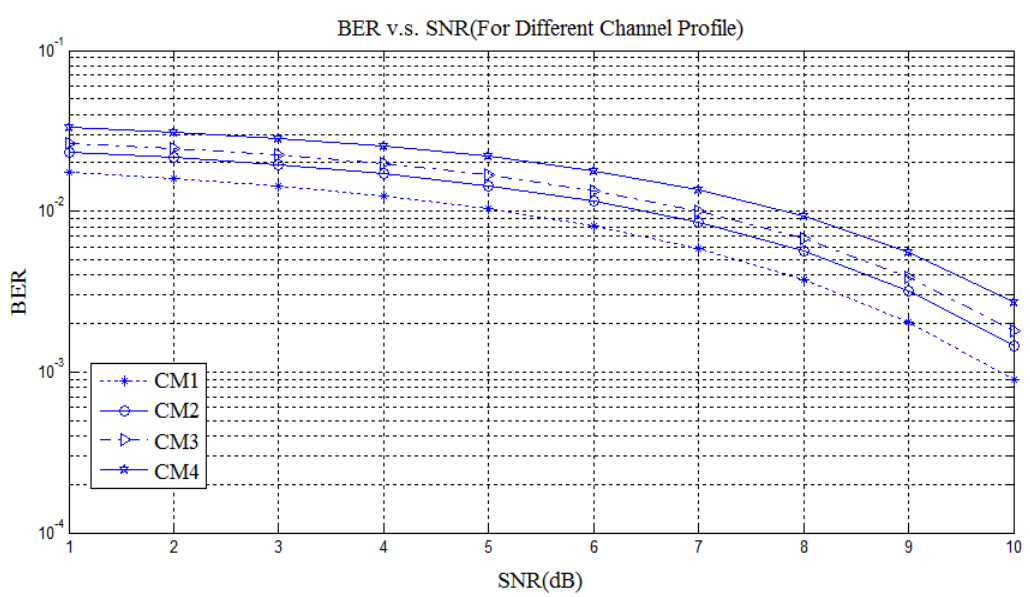

Fig.8. BER Performance of the IEEE 802.15.3a UWB Channel CM1, CM2, CM3, CM4 with best Modulation (BPSK) Scheme.

In the figure 8 we compare all channel profile on same platform with best modulation scheme which comes from previous result i.e. antipodal category (BPSK).

\section{Conclusions}

The major applications in the field of UWB are related to higher data transmission rate near about 300 to $500 \mathrm{Mbps}$ up to distance of 10ft. In such a higher data rate increment in the BER will not be better choice. Performance results show that the best modulation schemes in terms of ABER belongs to antipodal category. So best modulation scheme in favor of UWB belongs to antipodal (BPSK) category. One interesting research topic that can be extended from this work is to apply this method to any multipath fading models with other general random arrival process for clusters and rays, such as the IEEE 802.15.4a outdoor channel model. 


\section{References}

[1] A. F. Molisch, J. R. Foerster, and M. Pendergrass, "Channel models for ultrawideband personal area networks," IEEE Wireless Commun., vol. 10, pp. 14-21, Dec. 2003.

[2] J. Foerster, Ed., "Channel Modeling Sub-committee Report Final," IEEE, Document IEEE P802.1502/490r1-SG3a, 2003.

[3] A. A. M. Saleh and R. Valenzuela, “A statistical model for indoor multipath propagation,” IEEE J. Select. Areas. Commun., vol. SAC-5, no. 2, pp. 128-137, Feb. 1987.

[4] H. Liu, "Error performance of a pulse amplitude and position modulated ultra-wideband system over lognormal fading channels," IEEE Communication. Letter, vol. 7, pp. 531-533, Nov. 2003.

[5] Ashish K. Thakre, Amol I. Dhenge, "Selection of pulse for ultra wide band communication (UWB) system", International Journal of Advanced Research in Computer and Communication Engineering Vol. 1, Issue 9, November 2012.

[6] A.-R. Chadi, "Performance analysis of UWB systems over the IEEE802.15.3a channel model," IEEE Trans. Commun., vol. 59, no. 9, pp. 2377-2382, Sep. 2011.

[7] John G. Proakis, Masoud Salehi, Digital Communications, 5th ed. New York: McGraw-Hill, 2008, ch. 14.

[8] Huseyin Arslan, Zhi Ning Chen, Maria-Gabriella Di Benedetto, Ultra wideband wireless communication, 3rd ed. New John Wiley \& Sons Ltd, 2006, ch. 4.

[9] Tri Budi Santoso, "Final Report of Telecommunication Engineering Training Suzuki Laboratory, Mobile Communication Group Tokyo Institute of Technology, Japan, Differential PSK (DPSK) (5.2.8) pp: 274 278.

[10] N. C. Beaulieu, A. A. Abu-Dayya, and P. J. Mclane, "Estimating the distribution of a sum of independent lognormal random variable," IEEETrans. Commun., vol. 43, pp. 2869-2873, Dec. 1995.

[11] Ian Oppermann, Matti Hamalainen and Jari Iinatti, UWB Theory and Applications, 1st ed. New John Wiley \& Sons Ltd, 2004, ch. 4.

\section{Authors' Profiles}

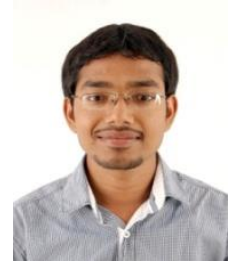

Sachin Taran (born Jan 24, 1986) is an Assistant Professor in Medicaps Institute of Science and Technology Indore. He has completed his Bachelor of Engineering from SATI Vidisha and Masters from IET DAVV Indore. He has qualified higher education entrance exam of India (GATE) many times. He has seven year work experience of teaching field. His research interest is Wireless Communication and Image Processing.

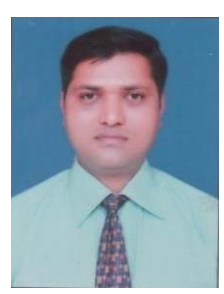

Dr. Dhiraj Nitnaware is an Assistant Professor in IET DAVV Indore. He has completed his Bachelor of Engineering from GEC Ujjain and Masters from SOI DAVV Indore. He completed his doctors from IET DAVV Indore. He has nine year work experience of teaching field and one year experience of industry. His research interest is Wireless Adhoc Network, Wireless Sensor Network and image processing. 